\title{
Modeling and simulation of phototransduction cascade in vertebrate rod photoreceptors
}

\author{
Guofeng Pan ${ }^{1}$, Jinglu Tan² and Ya Guo ${ }^{1,2^{*}}$
}

\begin{abstract}
Background: The activation of phototransduction cascade in rod photoreceptors has been well studied in literature, but there is a lack of a mature kinetic model structure covering both the activation and inactivation processes.

Methods: In this work, a kinetic model structure is developed to describe the major activation and inactivation processes in vertebrate rod photoreceptors with the electroretinogram (ERG) as output. Simulation was performed to validate developed model structure.

Results: The developed model structure could fit experimental data with small error.

Conclusions: The result indicated that the developed model structure could show the inactivation process of phototransduction cascades in the rod photoreceptors.
\end{abstract}

Keywords: Phototransduction, ERG a wave, Rod, Photoreceptor, Kinetic model structure

\section{Background}

When the molecules of rod photoreceptors, rhodopsin, absorb photons, a cascade of reactions are triggered [10, 13]. This eventually results in the decline of intracellular cGMP concentration and closure of $c G M P$ gated $\mathrm{Na}^{+}$/ $\mathrm{Ca}^{2+}$ ion channels in plasma membrane of rods, and leads to changes of electrical current and voltage [17]. The electrical current or voltage from the photoreceptors is an important part of electroretinogram (ERG), which is the summation of a few components of extracellular currents from different cells [13, 25, 28]. ERG has become an important diagnosis tool as the measurement standard protocols are developed and commercial instruments are available $[8,12,19,20,22]$. The response in the photoreceptor is very important to vision because it is the early stage of ERG and triggers latter vision activities $[2,5,7,16]$.

In literature, there are many efforts on modeling and simulation of phototransduction cascade in rods and ERG generation $[6,9,11,12,16,17,21,26,27]$. A recent

\footnotetext{
*Correspondence: guoy@missouri.edu

${ }^{1}$ Ministry of Education, Key Laboratory of Advanced Process Control for Light Industry, Jiangnan University, Wuxi 214122, China

2University of Missouri, Columbia, MO 65211, USA
}

review on modeling the retina in health, development and disease was well conducted by Roberts et al. [24]. Phenomenological analytical functions or stochastic events are often used to simulate the processes. These efforts have significantly contributed to the understanding of phototransduction cascade and vision system. However, there are limitations if they are applied for the estimation of reaction rates from measured ERG signal because the reaction rates are not explicit variables in phenomenological model structure and there are many difficulties (e.g. computation speed) in estimating model parameters from stochastic event models. The models in literature usually only focus on the activation processes. Estimation of reaction rates from measured ERG signal can be very useful for diagnosis; therefore, it is meaningful to develop a kinetic model structure with reaction rates as parameters for both the activation and inactivation processes. A kinetic model structure for this purpose should be fundamentally based on major reaction kinetics but not include too many reaction details; otherwise, the complexity from the details will incur difficulty in parameter estimation algorithm convergence and reduce computation speed. Currently, there is a lack of a mature kinetic model structure covering both the

(c) The Author(s). 2019 Open Access This article is distributed under the terms of the Creative Commons Attribution 4.0 International License (http://creativecommons.org/licenses/by/4.0/), which permits unrestricted use, distribution, and 
activation and inactivation processes. In this work, a kinetic model structure is developed, which included the major reactions phototransduction cascade. The capability of the model structure to represent the inactivation process has been demonstrated.

\section{Method}

Based on published work on the molecular mechanisms for phototransduction cascade in vertebrate rod photoreceptors, the involved major activation and inactivation biochemical reactions are summarized and the model structure is developed.

\section{Activation of rhodopsin}

A captured photon may set an inactivated rhodopsin $(R)$ to its activated state $\left(R^{*}\right)$ by isomerizing its chromophore from 11-cis to the all-trans form $[1,12,13]$. The production rate of $R^{*}$ is proportional to the concentration of $R$ and light intensity $u$, which can be represented by:

$$
R \stackrel{k_{1} u}{\rightarrow} R^{*}
$$

where $k_{1}$ is the activation rate of rhodopsin.

\section{Activation of G-protein (G-GDP) by activated rhodopsin}

Through diffusion on the disc membrane, $R^{*}$ and inactivated G-GDP interact, which results in a series of reactions including [12, 16]: (1) $R^{*}$ binds to G-GDP and $R^{*}$-G-GDP is formed, (2) GDP is released and $R^{*}$-G is formed, (3) GTP binds to $R^{*}-\mathrm{G}$ and $R^{*}-\mathrm{G}-\mathrm{GTP}$ is formed, (4) $R^{*}$ is released and G-GTP is formed, which then separates into submits $G_{\beta \gamma}$ and $G_{\alpha}{ }^{*}$-GTP. $G_{\alpha}{ }^{*}$-GTP is activated and can trigger further reactions. $R^{*}$ is not altered in the processes and serves as an enzyme. For simplification, $G$ is used to denote G-GDP and $G^{*}$ is used to denote the activated $\mathrm{G}_{\alpha}{ }^{*}$-GTP. It is not practical to include all the reaction details; otherwise, the complexity of the model structure will be dramatically increased. According to Michaelis-Menten kinetics, the intermediate reactions are simplified and these reactions can be approximately represented as:

$$
R^{*}+G \underset{k_{3}}{\stackrel{k_{2}}{\rightleftarrows}} C_{1} \stackrel{k_{4}}{\longrightarrow} R^{*}+G^{*}
$$

where $C_{1}$ is the intermediate complex formed by the binding of $R^{*}$ and $G$, and $k_{2}, k_{3}$, and $k_{4}$ are the reaction rate constants.

\section{Activation of phosphodiesterase (PDE) by activated $\mathrm{G}^{*}$}

Two $G^{*}$ units bind to the two inhibitory subunits of inactivated PDE (denoted as $E$ ) and activate the $\alpha$ and $\beta$ catalytic subunits of PDE and make PDE in active state $[1,12]$; however, the two PDE subunits seem to work independently. If $E^{*}$ is used to denote a single activated subunit of PDE, the activation process of PDE can thus be represented as [11]:

$$
G^{*}+E \stackrel{k_{5}}{\rightarrow} E^{*}
$$

where $k_{5}$ is the activation rate of $E$.

\section{Change of cyclic GMP (cGMP) concentration}

$E^{*}$ catalyzes the hydrolysis of $c G M P$ (denoted as $c G$ for conciseness) and produces GMP [23]. According to Michaelis-Menten kinetics, this process can be represented as:

$$
E^{*}+c G \underset{k_{7}}{\stackrel{k_{6}}{\rightleftarrows}} C_{2} \stackrel{k_{8}}{\rightarrow} E^{*}+G M P
$$

where $C_{2}$ is the intermediate complex formed by the binding of $E^{*}$ and $c G$, and $k_{6}, k_{7}$, and $k_{8}$ are the reaction rate constants.

\section{Inactivation of $\mathrm{E}^{*}$ and $\mathrm{G}^{*}$}

The GTP bound to $G^{*}$ in the complex of $E^{*}$ is hydrolyzed to GDP, which results in separation of the complex, production of PDE and $\mathrm{G}_{\alpha}$-GDP $\left(G_{r}\right)$, and inactivation of both $E^{*}$ and $G^{*}$. $G_{r}$ will finally convert to G-GDP $(G)[1,6]$. These processes can be represented as:

$$
\begin{aligned}
& E^{*} \stackrel{k_{9}}{\rightarrow} G_{r}+E \\
& G_{r} \stackrel{k_{10}}{\rightarrow} G
\end{aligned}
$$

where $k_{9}$ is the rate of $E^{*}$ hydroxylation and $k_{10}$ is the rate of $G_{r}$ converts to $G$.

\section{Channel activity, $\mathrm{R}^{*}$ inactivation, and cGMP restoration}

The decrease of $c G M P$ concentration as represented in Eq. (4) leads to closure of $c G M P$ gated channels and reduction of $\mathrm{Ca}^{2+}$ influx, and thus cytoplasmic $\mathrm{Ca}^{2+}$ concentration is dropped. If $\mathrm{Ca}$ is used to denote the cytoplasmic $\mathrm{Ca}^{2+}$ concentration, the drop of $\mathrm{Ca}^{2+}$ concentration can be represented as:

$$
\frac{d(C a)}{d t}=-k_{11}\left(c G_{0}^{n c G}-c G^{n c G}\right)
$$

where $c G_{0}$ is the concentration of $c G M P$ after dark-adaption, $c G$ is the current $c G M P$ concentration, $k_{11}$ is a constant, $n_{\mathrm{cG}}$ is the Hill coefficient that describes cGMP opening channel in a cooperative manner [1]. In this work, $n_{\mathrm{cG}}$ is set as 2 [12].

The reduced $\mathrm{Ca}^{2+}$ concentration causes recoverin (a guannylyl cyclase activation protein, GCAP) to release its $\mathrm{Ca}^{2+}$ and separate from rhodopsinkinase (RK). The increase of $\mathrm{Ca}^{2+}$ concentration is initiated by $\mathrm{Ca}^{2+}$ concentration drop from the dark-adapted value $C a_{0}$ and is 


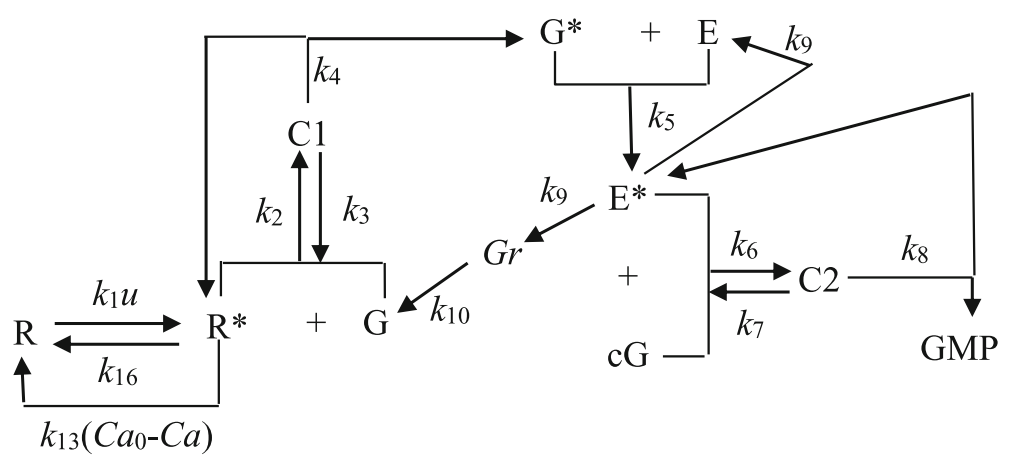

Fig. 1 Major chemical reaction transition diagram (R-rhodopsin, $R^{*}$-activated rhodopsin, G--G protein, $G^{*}$-- activated G protein, E--phosphodiesterase or PDE, E*-- a single activated subunit of PDE, CG--CGMP, C1 and C2--intermediate complexes. Mass diffusion processes. $u$ denotes light intensity. All the " $k$ "s are reaction rates. Typically, they have the unit $1 / \mathrm{s}$ (first reaction kinetics) or mol/s (second reaction kinetics) depending on the unit used for the concentrations)

assumed as proportional to the concentration drop, which implies that the concentration change can be represented as:

$$
\frac{d(C a)}{d t}=k_{12}\left(C a_{0}-C a\right)
$$

where $k_{12}$ is the rate that $C a$ is increased.

The free form of RK phosphorylizes $R^{*}$ and allows arrestin (Arr) to bind and inactivate $R^{*}$. The inactivation of $R^{*}$ in this way is mediated by $\mathrm{Ca}^{2+}$ and originates from the reduction of $\mathrm{Ca}^{2+}$ concentration. For simplicity, the inactivation of $R^{*}$ is assumed as proportional to the $\mathrm{Ca}^{2+}$ concentration drop from its dark-adapted value $\mathrm{Ca}_{0}$ and thus can be represented as:

$$
R^{*} \stackrel{k_{13}\left(\mathrm{Ca}_{0}-\mathrm{Ca}\right)}{\rightarrow} R
$$

where $k_{13}$ is the deviation rate of $R^{*}$ mediated by the reduction of $\mathrm{Ca}^{2+}$ concentration.

The $\mathrm{Ca}^{2+}$-free form of GCAP will bind to gyanylyl cyclase (GC) and turn on the enzymatic activity of $\mathrm{GC}$, which catalyzes the synthesis of $c G M P$ from GTP [16, 23]. According to Biernbaum and Bownds
[3], GTP concentration change only occurs when light intensity is stronger than the saturation level and much later than the GC catalyzed cGMP production. In this work, the concentration of GTP is thus considered as a constant. Again, this activity is initiated and mediated by $\mathrm{Ca}^{2+}$. For simplicity, the synthesis of cGMP is assumed as proportional to the $\mathrm{Ca}^{2+}$ concentration drop from its dark-adapted value $C a_{0}$ and the drop of $c G$ concentration from its dark-adapted value $c G_{0}$. It is thus the change of $c G$ concentration can be represented as:

$$
\frac{d(c G)}{d t}=k_{14}\left(C a_{0}-C a\right)\left(c G_{0}-c G\right)
$$

where $k_{14}$ is the synthesis rate of $c G M P$ mediated by the reduction of $\mathrm{Ca}^{2+}$ concentration.

Reduction of cGMP concentration will also cause cGMP buffer in the cytoplasm releasing free form of cGMP and the diffusion of cGMP under concentration gradient [23]. If these activities are assumed to proportional to the drop of cGMP concentration, the increase

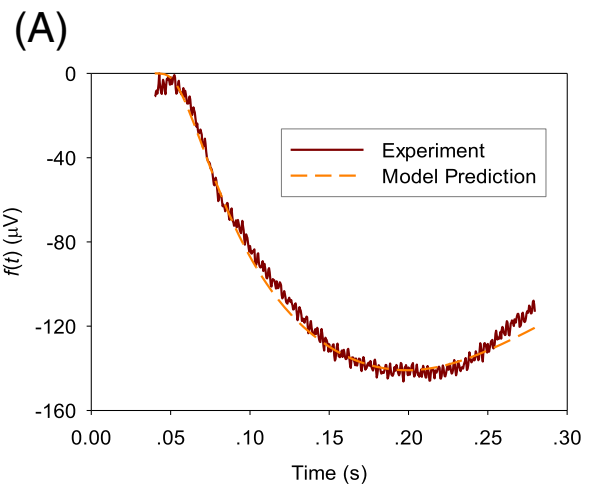

(B)

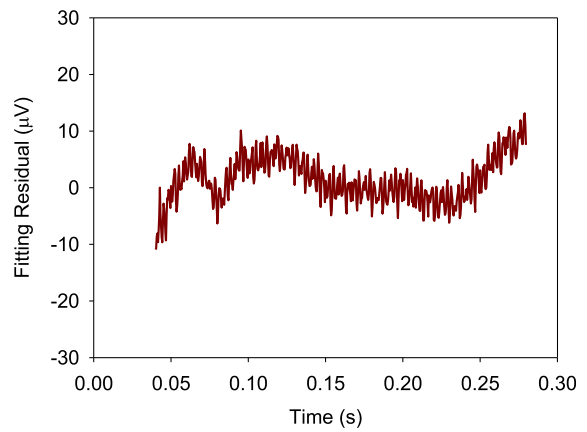

Fig. 2 A comparison of averaged value of measured ERG data $f(t)$ from healthy NOB1 mice with model prediction a and the fitting residual $\mathbf{b}$ 
(A)

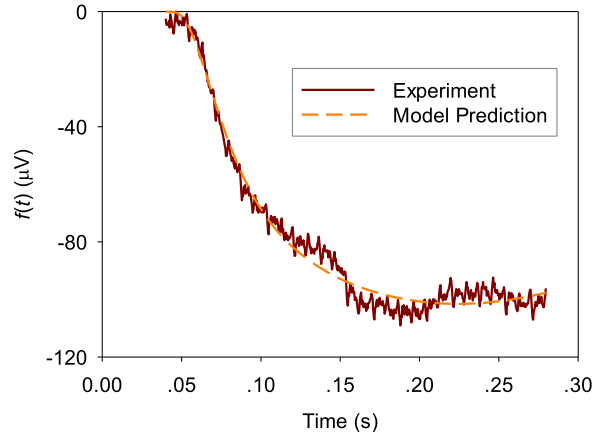

(B)

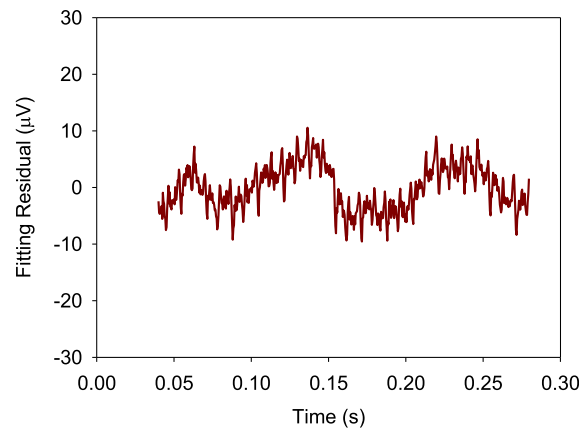

Fig. 3 A comparison of averaged value of measured ERG data $f(t)$ from photoreceptor-damaged NOB1 mice with model prediction a and the fitting residual $\mathbf{b}$

of free cGMP concentration in cytoplasm can be represented as:

$$
\frac{d(c G)}{d t}=k_{15}\left(c G_{0}-c G\right)
$$

where $k_{15}$ is a constant.

Independent of RK and Arr, R* may be inactivated in an abnormal and prolonged manner [12]. This process is represented as:

$$
R^{*} \stackrel{k_{16}}{\rightarrow} R
$$

where $k_{16}$ is the rate of $R^{*}$ deactivation that is not caused by the activities mediated by $\mathrm{Ca}^{2+}$ concentration change as in Eq. (9).

All major chemical reaction transitions are summarized in Fig. 1.

Let $x_{1}$ through $x_{8}$ denote the concentrations of $R^{*}, G^{*}$, $C_{1}, E^{*}, C_{2}, c G, G r$, and $C a^{2+}$ respectively. The concentration at the balance after dark-adaptation for $R, G, E, c G$, $C a$ are denoted as $R_{0}, G_{0}, E_{0}, c G_{0}, C a_{0}$, respectively. According to chemical reaction kinetics, reaction speed is usually proportional to species concentration or the probability that reactants meet each other, species concentration changes rate with time represented by the phototransduction cascade in Eqns. (1) through (12) can be written as:

$$
\begin{aligned}
\frac{d x_{1}}{d t}= & k_{1 u}\left(R_{0}-x_{1}-x_{3}\right)-k_{2} x_{1}\left(G_{0}-x_{3}-x_{2}-x_{4}-x_{5}-x_{7}\right) \\
& +k_{3} x_{3}+k_{4} x_{3}-k_{13}\left(C a_{0}-x_{8}\right) x_{1}-k_{16} x_{1}
\end{aligned}
$$

$$
\frac{d x_{2}}{d t}=k_{4} x_{3}-k_{5} x_{2}\left(E_{0}-x_{4}-x_{5}\right)
$$

$\frac{d x_{3}}{d t}=k_{2} x_{1}\left(G_{0}-x_{3}-x_{2}-x_{4}-x_{5}-x_{7}\right)-k_{3} x_{3}-k_{4} x_{3}$

$\frac{d x_{4}}{d t}=k_{5} x_{2}\left(E_{0}-x_{4}-x_{5}\right)-k_{6} x_{4} x_{6}+k_{7} x_{5}$ $+k_{8} x_{5}-k_{9} x_{4}$

$$
\frac{d x_{5}}{d t}=k_{6} x_{4} x_{6}-k_{7} x_{5}-k_{8} x_{5}
$$

\section{(A)}

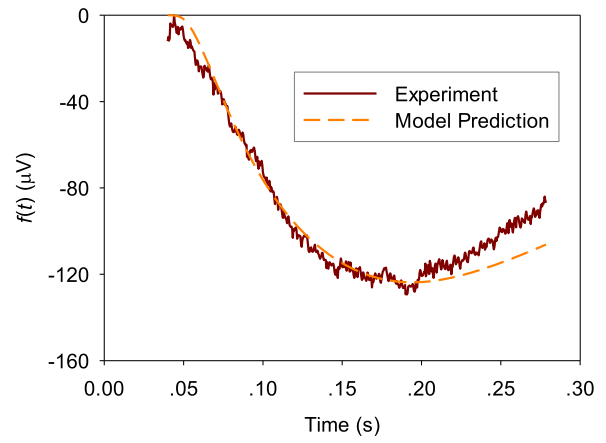

(B)

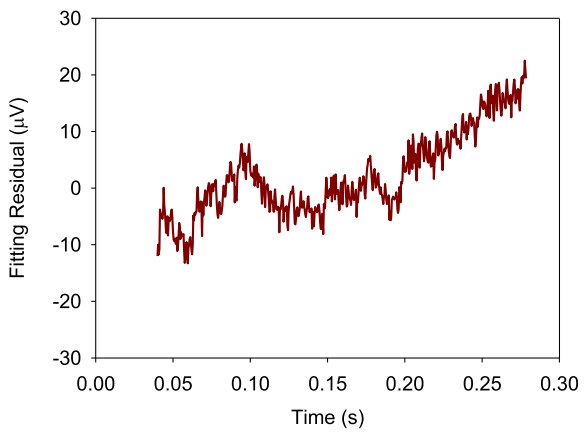

Fig. 4 A comparison of averaged value of measured ERG data $f(t)$ from wild-type mice with model prediction a and the fitting residual $\mathbf{b}$ 
Table 1 Means of $k_{1 u}$ to $k_{17}$ for the three groups of mice

\begin{tabular}{|c|c|c|}
\hline Model Parameter (or feature) & Groups & Values \\
\hline \multirow[t]{3}{*}{$k_{1 u}$} & NOB & 0.6717 \\
\hline & NOB with Drug & 0.5488 \\
\hline & Wild & 0.7487 \\
\hline \multirow[t]{3}{*}{$k_{2}$} & NOB & 17.9455 \\
\hline & NOB with Drug & 27.5449 \\
\hline & Wild & 17.5549 \\
\hline \multirow[t]{3}{*}{$k_{3}$} & NOB & 18.2341 \\
\hline & NOB with Drug & 14.4897 \\
\hline & Wild & 17.4280 \\
\hline \multirow[t]{3}{*}{$k_{4}$} & NOB & 5847.3409 \\
\hline & NOB with Drug & 3997.7881 \\
\hline & Wild & 5723.8125 \\
\hline \multirow[t]{3}{*}{$k_{5}$} & NOB & 52.3225 \\
\hline & NOB with Drug & 40.9749 \\
\hline & Wild & 51.0795 \\
\hline \multirow[t]{3}{*}{$k_{6}$} & NOB & 0.2329 \\
\hline & NOB with Drug & 2.3551 \\
\hline & Wild & 0.2336 \\
\hline \multirow[t]{3}{*}{$k_{7}$} & NOB & 45.6849 \\
\hline & NOB with Drug & 34.2112 \\
\hline & Wild & 45.7306 \\
\hline \multirow[t]{3}{*}{$k_{8}$} & NOB & 36.8285 \\
\hline & NOB with Drug & 24.9522 \\
\hline & Wild & 36.2993 \\
\hline \multirow[t]{3}{*}{$k_{9}$} & NOB & 6.1385 \\
\hline & NOB with Drug & 5.4685 \\
\hline & Wild & 6.0209 \\
\hline \multirow[t]{3}{*}{$k_{10}$} & NOB & 1.2880 \\
\hline & NOB with Drug & 1.2817 \\
\hline & Wild & 1.2676 \\
\hline \multirow[t]{3}{*}{$k_{11}$} & NOB & 52.8416 \\
\hline & NOB with Drug & 35.9586 \\
\hline & Wild & 53.0859 \\
\hline \multirow[t]{3}{*}{$k_{12}$} & NOB & 31.2042 \\
\hline & NOB with Drug & 27.3478 \\
\hline & Wild & 28.8673 \\
\hline \multirow[t]{3}{*}{$k_{13}$} & NOB & 79.5587 \\
\hline & NOB with Drug & 56.7860 \\
\hline & Wild & 77.7604 \\
\hline \multirow[t]{3}{*}{$k_{14}$} & NOB & 14.9012 \\
\hline & NOB with Drug & 2.1693 \\
\hline & Wild & 14.7936 \\
\hline \multirow[t]{2}{*}{$k_{15}$} & NOB & 11.0894 \\
\hline & NOB with Drug & 9.0645 \\
\hline
\end{tabular}

Table 1 Means of $k_{1 u}$ to $k_{17}$ for the three groups of mice (Continued)

\begin{tabular}{lll}
\hline Model Parameter (or feature) & Groups & Values \\
\hline \multirow{4}{*}{$k_{16}$} & Wild & 10.6546 \\
& NOB & 6.5656 \\
& NOB with Drug & 3.1117 \\
& Wild & 5.5993 \\
$k_{17}$ & NOB & 1649.2012 \\
& NOB with Drug & 614.8300 \\
& Wild & 2227.1000 \\
\hline
\end{tabular}

$$
\begin{aligned}
\frac{d x_{6}}{d t}= & -k_{6} x_{4} x_{6}+k_{7} x_{5}+k_{14}\left(C a_{0}-x_{8}\right)\left(c G_{0}-x_{6}\right) \\
& +k_{15}\left(c G_{0}-x_{6}\right) \\
\frac{d x_{7}}{d t}= & k_{9} x_{4}-k_{10} x_{7} \\
\frac{d x_{8}}{d t}= & -k_{11}\left(c G_{0}^{n c G}-x_{6}^{n c G}\right)+k_{12}\left(C a_{0}-x_{8}\right)
\end{aligned}
$$

Changes of cGMP concentration $\left(x_{6}\right)$ lead to closing or opening of cGMP-gated ion channels and changes in the circulating current between the outer and inner segments of photoreceptor (Lam and Pugh, 2004; [16]). The deviation of current from the dark-value is proportional to the changes of closing and opening of cGMP-gated channels. The recorded electrical voltage can be represented as:

$$
f(t)=k_{17}\left(c G_{0}^{n c G}-x_{6}^{n c G}\right)
$$

where $f(t)$ is the measured ERG signal caused by light stimulation, $k_{17}$ is a gain factor accounting for the number of photoreceptors and instrumentation gain (Because the produced current is proportional to the number of photoreceptors and instrumentation gain, the two factors will appear as product, which is merged into one term and cannot be separated.).

\section{Results}

ERG data were reproduced from the publication of $\mathrm{Lu}$ et al. [16] and Lu [15]. ERG were recorded from three wild-type mice (61-day-oldm males), three NOB1 mice (healthy, 61-day-old), and three NOB1 mice with drug treatment (N-methy-N-nitrosourea). The bipolar cells of NOB1 mice were genetically disabled to suppress ERG b-wave. Details about the experiments can be found in $\mathrm{Lu}$ et al. [16]. The Levenberg-Marquardt method was used to estimate the model parameters $\left(k_{1}-k_{17}\right)$ in this work, which was adopted from the Matlab codes in the appendix of Lu [15]. In the Levenberg-Marquardt method, the increment of each model parameter was computed according to the Jacobian matrix of partial derivatives of $f(t)$ 
with respect to $\left(k_{1}-k_{17}\right)$ evaluated at all data points, the error between the model prediction and experimental data, and a damping factor for improving algorithm convergence. The algorithm of the Levenberg-Marquardt method can be found in Marquardt [18], Levenberg [14], Constantinides and Mostoufi [4]. The Runge-Kutta algorithm was used to integrate the differentiation equations. Both the Runge-Kutta and the Levenberg-Marquardt were programmed in Matlab. The model parameters and the total or dark concentrations in Eqns. (13) through (21) can always be normalized by redefining the state variables and maintaining a constant $R_{0}$. It is thus the ratios of the state variables define the shape of ERG. The ratios for the initial values for $R_{0}, G_{0}, E_{0}, c G_{0}$, and $C_{0}$ were set as $50,5,0.5,4$, and 0.22 , respectively. The initial values for the state variables of $x_{1}$ through $x_{8}$ were set as $\left[\begin{array}{llllllllll}0 & 0 & 0 & 0 & 0 & \mathrm{cG}_{0} & 0 & \mathrm{Ca}_{0}\end{array}\right]$ since fully dark adaptation was applied before measurements. In this work, the values of total or dark concentrations $\left(G_{0}\right.$, $E_{0}, c G_{0}$, and $C a_{0}$ ) were also optimized in the algorithm while $R_{0}$ was maintained as a constant for all the groups. By doing this, the number of unknown parameters was reduced by 1 , which was significant to improving computation speed and algorithm convergence. Accordingly, the estimated model parameters were effective rates. The constant $R_{0}$ value served as a reference and made the estimated effective rates comparable and could be used for classification as demonstrated in this work.

Although NOB1mouse and a chemical technique [16] were used to reduce ERG b-wave, part of the b-wave and/or other ERG components from cells other than rod photoreceptors still existed. Because the response of photoreceptors is the early part of ERG, only the initial data segment from the beginning of light stimulus to a moment slightly after ERG trough was used to estimate model parameters. In this way, the effect of ERG components from other cells was reduced since the proposed model structure was only for the phototransduction cascade in rod photoreceptors.

Figures 2, 3, and 4 show the comparisons of the averaged value of measured ERG data with model predictions and fitting residual for healthy NOB1, photoreceptor-damaged, and wild-type mice till $0.28 \mathrm{~s}$, respectively. As shown in these figures, the model prediction could largely fit the initial stage of ERG, which is mainly from the rod photoreceptors. The relative fitting error for each of the three individual groups is less than $10 \%$ and the averaged value of relative errors for all the three groups of mice is $6.8 \%$.

The mean value of $k_{1 u}$ to $k_{17}$ for the three groups of mice are shown in Table 1.

In order to verify the capability of the model structure to represent the inactivation process of rod phototransduction cascade, the parameters estimated from the

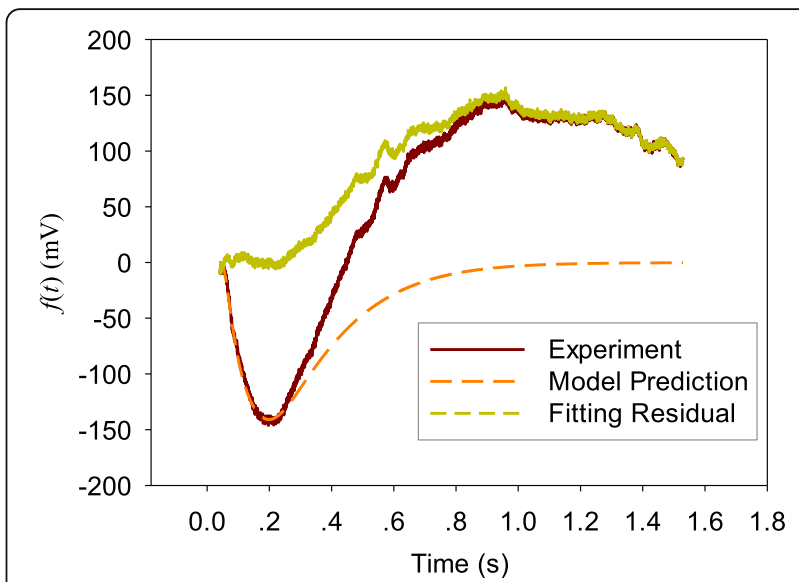

Fig. 5 Simulation of rod photoreceptor response $f(t)$ of a healthy NOB1 mouse for a longer time

initial part of the ERG data were used to simulate the photoreceptor response for a much longer time. Figures 5, 6, and 7 show the simulation results, measured ERG data, and the difference between the simulated results and ERG data (fitting residual) for the three types of mice (healthy NOB1, photoreceptor-damaged NOB1, and wild-type), respectively. The simulated response from photoreceptors can match the initial part of ERGs and return the dark-adapted value after the stimulation light is turned off for a while, which agree with the long term behavior of rods and ERGs without the responses from other cells like bipolar cell [21]. The fitting residuals in the three figures can serve as predictions of the ERG components from cells other than rod photoreceptors.

\section{Discussions}

Observations of Figs. 2, 3 and 4 reveal that the fitting errors mainly come from two sources. The first one is the

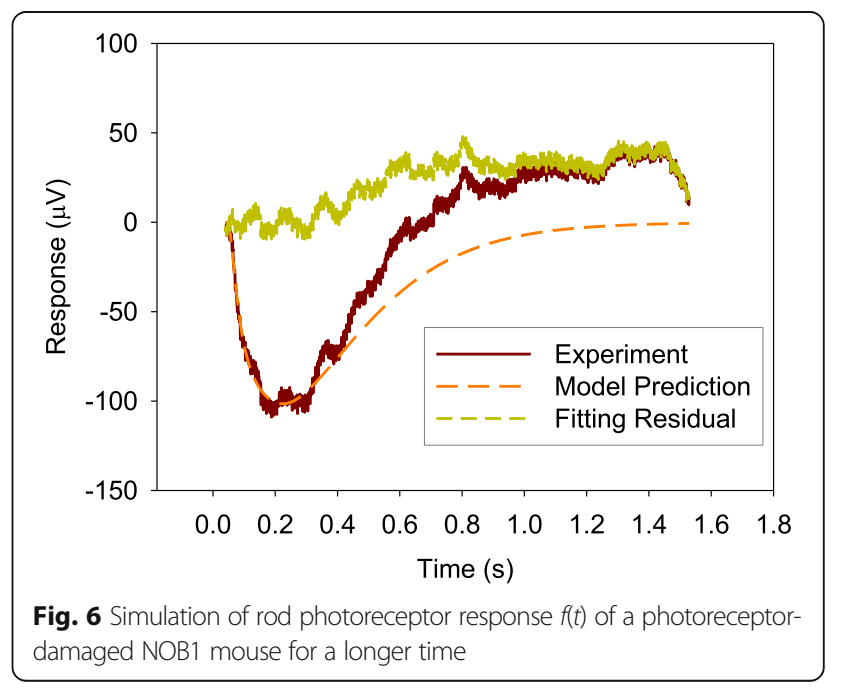




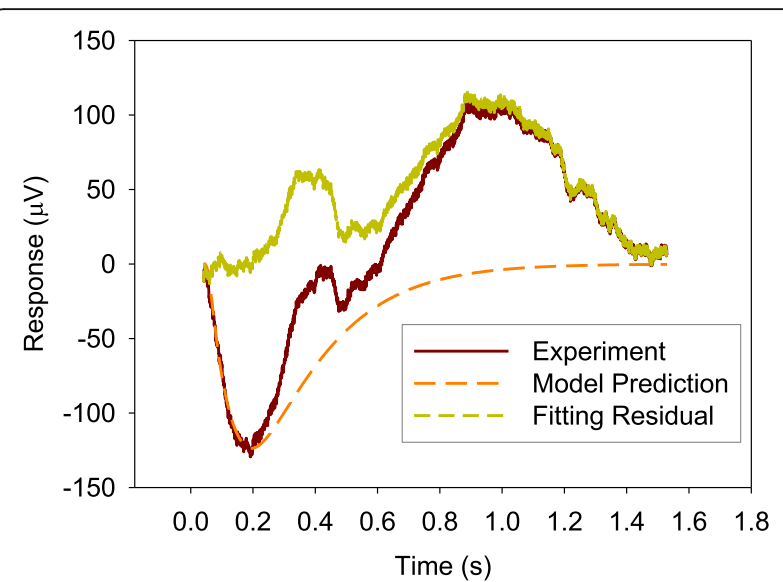

Fig. 7 Simulation of rod photoreceptor response $f(t)$ of a wild-type mouse for a longer time

high frequency noise on top of the signal, the other one is the discrepancy between experimental data and model prediction after the lowest trough of ERG. Because the existence of b-wave (generate later than the photoreceptor a-wave) at some degree makes ERG increase faster and the model structure does not cover the generation of ERG b-wave, the fitting error is larger at the later stage (after $0.2 \mathrm{~s}$ ). The experimental data are bigger than model prediction for healthy NOB1 and wild-type subjects after the lowest trough of the ERGs. The fitting residual for wild-type group is stronger than the healthy NOB1 group, which suggests that the b-wave in ERG of the wild type group has more weight.

It is worth mentioning that the estimated reaction rates are effective rates because the initial concentrations were normalized. They are not comparable to the commonly reported pseudo first-order rates, but this does not affect the capability of the model structure to fit experimental data. In future research, further experiments including more subjects under other experimental conditions are needed to validate the developed model structure and compared model parameter changes with retina diseases.

\section{Conclusions}

In this work, a kinetic model structure is developed to describe the major reactions of activation and inactivation of phototrasduction cascade in rods. Measured ERGs from three groups of mice (NOB1, photo-receptor damaged NOB1, and Wild-type) are used to validate the model structure. The developed model structure could fit experimental data with small error. The developed model structure can represent both the activation and inactivation activities in rod phototransductions. In future research, more effort is needed to validate and establish the developed model structure.

\section{Abbreviations}

cGMP: cyclic guanosine monophosphate; ERG: Electroretinogram; G-GDP: Gprotein; PDE: Phosphodiesterase; R: Rhodopsin; R*: activated rhodopsin

\section{Acknowledgements \\ Not applicable.}

\section{Funding}

This project is partially supported by the National Natural Science Foundation of China (No: 31771680, used in data analysis), the Fundamental Research Funds for the Central Universities of China (No: JUSRP51730A, used in data interpretation), the 111 Project (B12018, used in data interpretation), and the Research Funds for New Faculty in Jiangnan University (used in manuscript writing).

\section{Availability of data and materials}

All the data supporting our findings are available through email request from the corresponding author.

\section{Authors' contributions}

YG and JT conceived the idea. YG and PG did the modeling and data analysis. All the authors participated in the manuscript writing, and have read and approved the manuscript.

Ethics approval and consent to participate

Not applicable.

\section{Consent for publication}

Not applicable.

\section{Competing interests}

The authors declare that they have no competing interests.

\section{Publisher's Note}

Springer Nature remains neutral with regard to jurisdictional claims in published maps and institutional affiliations.

Received: 26 November 2018 Accepted: 24 January 2019

Published online: 20 February 2019

\section{References}

1. Arshavsky WY, Lamb TD, Pugh EN. G Proteins and Phototransduction. Annual Review of Physiology. 2002;64,153-87.

2. Armington JC. The electroretinogram (pp. 275-278). New York: Academic Press; 1974

3. Biernbaum MS, Bownds MD. Light-induced Changes in GTP and ATP in Frog Rod Photorecepters. Journal of General Physiology. 1985;85:107-121.

4. Constantinides A, Mostoufi N. Numerical methods for chemical engineers with MATLAB applications, vol. 443. Upper Saddle River, NJ: Prentice Hall PTR; 1999.

5. Einthoven W, Jolly WA. The form and magnitude of the electrical response of the eye to stimulation by light at various intensities. Exp Physiol. 1908; 1(4):373-416.

6. Felber S, Breuer HP, Petruccione F, Honerkamp J, Hofmann KP. Stochastic simulation of the transducinGTPase cycle. Biophys J. 1996:71(6):3051-63.

7. Granit R. The components of the retinal action potential in mammals and their relation to the discharge in the optic nerve. J Physiol. 1933; 77(3):207-39.

8. Karaśkiewicz J, Drobek-Słowik M, Lubiński W. Pattern electroretinogram (PERG) in the early diagnosis of normal-tension preperimetric glaucoma: a case report. Doc Ophthalmol. 2014;128(1):53-8.

9. Keener, J. \& Sneyd, J. Mathematical Physiology II: Systems Physiology. New York: Springer; 2009.

10. Laitko U, Hofmann KP. A model for the recovery kinetics of rod phototransduction, based on the enzymatic deactivation of rhodopsin. Biophys J. 1998;74(2):803-15.

11. Lamb TD. Gain and kinetics of activation in the G-protein cascade of phototransduction. Proc Natl Acad Sci. 1996;93(2):566-70.

12. Lamb TD, Pugh EN. A quantitative account of the activation steps involved in phototransduction in amphibian photoreceptors. J Physiol. 1992:449(1): 719-58. 
13. Lamb TD, Pugh EN Jr. Dark adaptation and the retinoid cycle of vision. Prog Retin Eye Res. 2004;23:307-80.

14. Levenberg K. A method for the solution of certain problems in least squares. Q Appl Math. 1944;2:164-8.

15. Lu L. Modeling of phototransduction in vision systems (master thesis, University of Missouri--Columbia); 2007.

16. Lu L, Lei B, Guo Y, Tan J. Modeling of the phototransduction cascade in vertebrate rod photoreceptor. In Biomedical Engineering and Informatics (BMEl), 2010 3rd International Conference on (Vol. 3, pp. 1221-1228). Yantai: IEEE; 2010.

17. Mahroo OA, Ban VS, Bussmann BM, Copley HC, Hammond CJ, Lamb TD. Modelling the initial phase of the human rod photoreceptor response to the onset of steady illumination. Doc Ophthalmol. 2012;124(2):125-31.

18. Marquardt DW. An algorithm for least-squares estimation of nonlinear parameters. J. Soc. Ind. Appl. Math. 1963;11(2):431-41.

19. Marr BP, Singh AD. Retinoblastoma: evaluation and diagnosis. In: Clinical ophthalmic oncology: Springer Berlin Heidelberg; 2015. p. 1-11.

20. Nair SS, Paul Joseph K. Wavelet based electroretinographic signal analysis for diagnosis. Biomed Signal Process Control. 2014;9:37-44.

21. Nikonov S, Engheta N, Pugh EN. Kinetics of recovery of the dark-adapted salamander rod photoresponse. J. Gen. Physiol. 1998;111(1):7-37.

22. Praidou A, Hagan R, Newman W, Chandna A. Early diagnosis of Stargardt disease with multifocal electroretinogram in children. Int Ophthalmol. 2014; 34(3):613-21.

23. Pugh EN Jr., Lamb TD. Phototransduction and Vertebrate Rods and Cones: Molecular Mechanisms of Amplification, Recovery and Light Adaptation. Molecular Mechanisms in Visual Transduction. ed. Stavenga WJ, de Grip WJ, Pugh EN Jr., Amsterdam; New York; Elsevier. 2000;183-255.

24. Roberts PA, Gaffney EA, Luthert PJ, Foss AJE, Byrne HM. Mathematical and computational models of the retina in health, development and disease. Prog Retin Eye Res. 2016;53:48-69.

25. Sneyd J, Tranchina D. Phototransduction in cones: an inverse problem in enzyme kinetics. Bull Math Biol. 1989;51:749-84.

26. Song Z, Coca D, Billings S, Postma M, Hardie RC. Biophysical Modeling of a Drosophila Photoreceptor, Neural Information Processing. Eds. Juusola M, Leung CS, Lee M, Chan JH. Berlin: Springer. 2009:5863:57-71.

27. Song Z, Postma M, Billings S, Coca D, Hardie R, Juusola M. Stochastic, adaptive sampling of information by microvilli in Fly photoreceptors. Curr Biol. 2012;22:1371-80.

28. Tranchina D, Sneyd J, Cadenas ID. Light adaptation in turtle cones. Testing and analysis of a model for phototransduction. Biophys J. 1991;60:217-37.

Ready to submit your research? Choose BMC and benefit from:

- fast, convenient online submission

- thorough peer review by experienced researchers in your field

- rapid publication on acceptance

- support for research data, including large and complex data types

- gold Open Access which fosters wider collaboration and increased citations

- maximum visibility for your research: over $100 \mathrm{M}$ website views per year

At $\mathrm{BMC}$, research is always in progress.

Learn more biomedcentral.com/submissions 